\title{
Recommendations with a Nudge
}

\author{
Randi Karlsen * (i) and Anders Andersen $(\mathbb{D}$ \\ Department of Computer Science, UiT The Arctic University of Norway, 9037 Tromsø, Norway; \\ Anders.Andersen@uit.no \\ * Correspondence: Randi.Karlsen@uit.no; Tel.: +47-77644040
}

Received: 12 April 2019; Accepted: 7 June 2019; Published: 13 June 2019

check for updates

\begin{abstract}
In areas such as health, environment, and energy consumption, there is a need to do better. A common goal in society is to get people to behave in ways that are sustainable for the environment or support a healthier lifestyle. Nudging is a term known from economics and political theory, for influencing decisions and behavior using suggestions, positive reinforcement, and other non-coercive means. With the extensive use of digital devices, nudging within a digital environment (known as digital nudging) has great potential. We introduce smart nudging, where the guidance of user behavior is presented through digital nudges tailored to be relevant to the current situation of each individual user. The ethics of smart nudging and the transparency of nudging is also discussed. We see a smart nudge as a recommendation to the user, followed by information that both motivates and helps the user choose the suggested behavior. This paper describes such nudgy recommendations, the design of a smart nudge, and an architecture for a smart nudging system. We compare smart nudging to traditional models for recommender systems, and we describe and discuss tools (or approaches) for nudge design. We discuss the challenges of designing personalized smart nudges that evolve and adapt according to the user's reactions to the previous nudging and possible behavioral change of the user.
\end{abstract}

Keywords: digital nudging; smart nudging; behavioral change; nudge-driven filtering; personalization; situation-awareness; nudge design; behavior recommendation

\section{Introduction}

Nudging is a term from economics and political theory for influencing decisions and behavior using suggestions, positive reinforcement, and other non-coercive means, to achieve socially desirable outcomes. The term nudge was first used in [1], where it was defined as "... any aspect of the choice architecture that alters people's behavior predictably without forbidding any options or significantly changing their economic incentives." Choice architecture refers to the "environment in which individuals make choices". The authors of [1] also stated that: "To count as a mere nudge, the intervention must be easy and cheap to avoid. Nudges are not mandating. Putting the fruit at eye level counts as a nudge. Banning junk food does not."

Nudges aim to influence people's behavior towards decisions that are beneficial for society, but usually also in the individual's long-term interest [1]. Nudging can, for example, encourage healthier or environmentally friendlier behavior. Since the goal is to change the behavior of users, the ethics of nudging should be considered when implementing nudging [2].

We aim at a system that provides suggestions (or recommendations) that, in some respects, are outside the users' primary interests or requirements. The nudging system monitors user activities and interests, and suggests, based on a nudging goal (e.g., making greener transportation choices), activities to the user that goes beyond the user's typical behavior. We are using knowledge about the user and the current situation to change the behavior of the user for a greater common good. 
Thus, a nudgy recommendation is not about providing the user with a suggestion that is well within the interest field of the user, but rather about making the user stretch, to achieve something in line with the nudging goal. Such recommendations typically challenge users to choose behavior that is, e.g. healthier or more environmentally friendly then they would otherwise do. The nudging goal might not match the original interests or requirements of the user. However, the goal is to change the behavior of the user, and maybe also the user's interests or requirements.

We use the term nudgy recommendations for non-coercive recommendations where the goal is to change the behavior of the user for the common good. To be effective, nudgy recommendations should be personalized and match the current situation of the user. Knowledge about the user and her situation may contribute to relevant nudges that are found useful and, therefore, might be followed by the user. Such knowledge is based on collecting a wide range of data, analyzing this in the context of the user, designing a personalized nudge, before it is used to inform and nudge the user. Through information collection and integration from multiple data sources, we seek to present people with information relevant to their decision makings, such as bus and train schedules, real-time traffic, congestions, road and footpath conditions, weather and air pollution level, and more.

The recommendations are provided in a complex environment, combining data from a wide variety of sources, constantly updating the user profile to reflect the user's responses to nudging, and tuning the nudges to improve the likelihood of a positive user response.

Back-end processing tasks can perform simple data integration and analysis or more complex data mining or machine learning based analysis. Some back-end processing tasks may produce results that are ready to be used to inform and nudge the user directly, while other back-end processing tasks produce a pre-processed result that needs further processing at the edge (e.g., at the user's mobile device).

We find that the final processing of a nudge normally happens at the edge. The reason for this is that the pre-processed data are combined with local, fresh, and possibly sensitive and private data on the user's smartphone. Such data include the user's calendar, current position, user profile, and current preferences.

This paper presents the concept of smart nudging as a personalized and context-sensitive recommender system using positive reinforcement to alter the behavior of people to a common good. The eight steps for designing a smart nudge are discussed, and an architecture for such a system is presented.

\section{Smart Nudging}

In this paper, we introduce the term smart nudging for digital nudging matching the current situation of the user. The nudge should both inform and motivate the user to choose the suggested activity or item. Digital nudging is described as a "subtle form of using design, information, and interaction elements to guide user behavior in digital environments, without restricting the individual's freedom of choice" [3]. We define smart nudging as digital nudging, where the guidance of user behavior is tailored to be relevant to the current situation of each individual user.

Tailoring of smart nudges is done through personalization and context-awareness, where knowledge about the user and her situation is central. Such knowledge is based on collecting a wide range of information, creating a user profile and analyzing information in the context of the user, before a personalized nudge is designed. We believe that a tailored nudge is more likely to succeed, i.e., being accepted and followed by the user, compared to non-tailored nudges.

Nudging people to a behavioral change represents a moving target. The goal is to nudge people to behave better, according to a nudging goal. To tailor nudges, user behavior must be monitored to determine the normal behavior of the user and how this behavior may change over time. As the behavior change, nudging should adapt to target the next level of desired behavior. If nudging does not affect user behavior, this is detected through monitoring, and the nudges should be adapted, 
for example by changing the timing, aimed behavior, information supporting the nudge and/or the presentation of the nudge.

\subsection{Designing a Smart Nudge}

Designing a smart nudge is illustrated in Figure 1, where a number of steps are identified. These design steps are inspired by Schneider et al. [4], who described the design of digital nudging. However, since our concern is smart nudging, we have added and altered steps compared to Schneider et al. [4]. In this paper, we focus on a use-case where the nudging goal is "choosing more environmentally friendly Transportation". However, steps for designing a smart nudge and the architecture for a nudging system (described in Section 3.2) is general and not restricted to this specific goal.

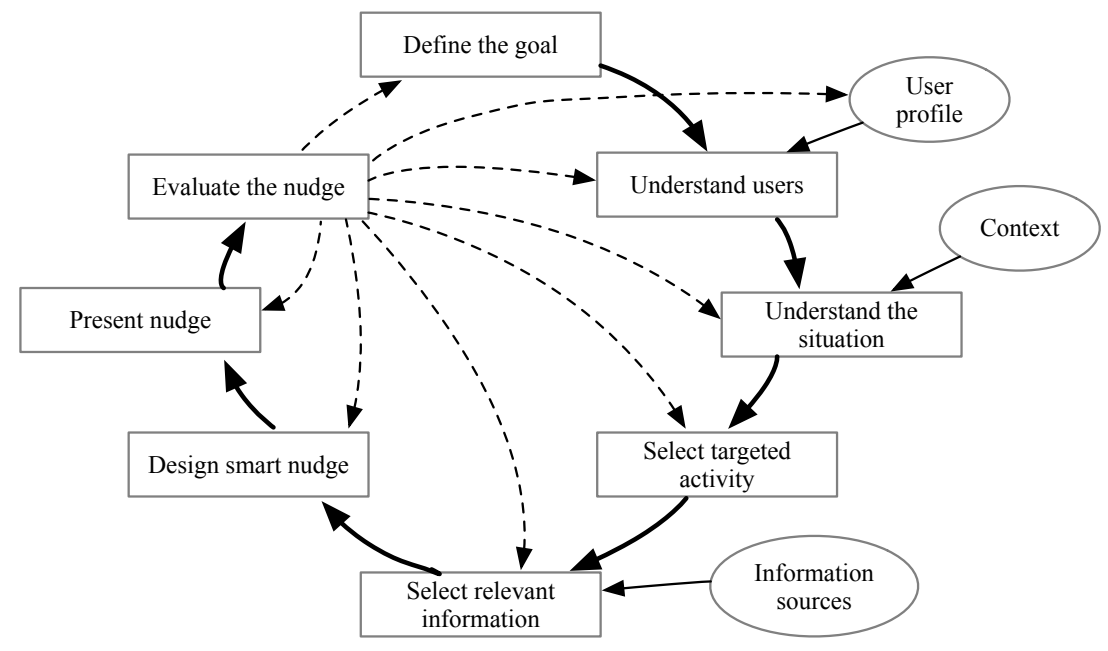

Figure 1. Designing a smart nudge.

Step 1: Define the goal. Here, the nudging goal is determined. This step is the overall goal for the nudgy recommendations and can be seen as the "greater good" that is to be achieved through nudging. Examples of nudging goals are a healthier lifestyle or using more environmentally friendly transportation.

Step 2: Understand the users. This step involves both understanding users in general, such as understanding the psychological effects that subconsciously influence people's behavior and decision making, but also understanding the specific user so that a personalized nudge (i.e., a smart nudge) can be designed.

Step 3: Understand the situation. This step is about understanding the context for the decision making, such as time of day, location, weather conditions, road conditions, and destination (an upcoming meeting may determine that). The situation influences which transportation choices are relevant for the specific user.

Step 4: Select the targeted activity. The nudging goal determines a set of relevant activities that can be suggested for the user. In this step, one of these activities is selected as the one for the user.

Step 5: Select relevant information. Here, the information used for designing the nudge is collected. The information comes from a number of different sources and may include weather information, bus routes and time tables, road and footpath conditions, and much more. The information is used for informing the user about the targeted activity, and gently pushes the user towards reacting positively to the nudge.

Step 6: Design the nudge. When designing the nudge, the selected activity is combined with relevant information so that the recommended activity can be presented using positive reinforcement and non-coercive means. The nudge will both provide practical information (such as bus routes and next departure time), making it convenient to choose the suggested activity, and motivating information that gently pushes towards the goal. 
Step 7: Present the nudge. After the nudge is designed, it is presented to the user in a timely manner and a suitable form that reaches the user at the time when a decision is to be made.

Step 8: Evaluate the nudge. The nudge presented to the user is evaluated according to how the user receives it. If the user follows the nudge, by selecting the recommended activity, the nudge is considered to be successful. The chosen activity is determined through the monitoring of the user or direct user feedback. Whether a nudge is successful or not can potentially influence all the other steps in the design process. The nudge and the chosen activity are recorded and can contribute to updating the user profile concerning preferred behavior. If a nudge is followed and this is a trend, it provides knowledge on what is an acceptable nudge for the user. If a nudge is not followed, this might indicate that upcoming nudges should adapt by, for example, target a different activity, be designed differently using other accompanying information, or be presented in a timelier manner. Evaluation of nudges can even lead to an evaluation of the nudging goal, considering whether the goal itself should be adjusted.

\subsection{Nudging for Green Transportation Choices}

Green transportation choices are about choosing environmentally friendly transportation means. Urban challenges of increased traffic, congestion, and air and noise pollution have become serious issues that need to be addressed [5]. These local challenges also have an impact on global scale issues, including climate changes and global warming, increased health problems, and bottlenecks in logistics chains. Urban mobility has to be rethought by making alternatives to private car usage more attractive [5]. Alternatives include walking, cycling, public transportation, and the usage of motorbikes and scooters. Another approach is to optimize and limit the usage of private cars through carpooling and incentive parking (park and ride) facilities.

Environmental friendliness can be placed on a scale, and the goal of nudging is to influence the user to make transportation choices that are more environmentally friendly than the default transportation choice for that specific person. Thus, for a person regularly driving to work with a private car, choosing the bus is a greener transportation choice, while choosing the bike or walking is even better. Table 1 gives examples of different types of transportation, a measure of environmental friendliness, and their discouraging and encouraging factors.

The quantification of environmental friendliness is complicated, and the EF measures given in Table 1 must be seen as approximations. For example, estimating environmental friendliness of vehicles are complex and may be based on a number of parameters, including the number of passengers, engine type (e.g., electric, gasoline, and diesel) and size [6], tire type (e.g., summer, winter, and studded), travel distance and travel time.

Table 1. Environmental friendliness (EF) and discouraging and encouraging factors for different types of transportation. Larger EF means environmentally friendlier type of transportation (from [7]).

\begin{tabular}{llll}
\hline Type & EF & Discouraging Factors & Encouraging Factors \\
\hline Car & & Economy (toll, parking, gas), traffic jam & Convenience \\
Carpool & & Inconvenience, traffic jam & Economy, social \\
Bus & Schedule, traffic jam & Economy, priority in traffic \\
Bike & Time, effort, exposed to conditions & Economy, health, pleasure \\
Walk & Time, effort, exposed to conditions & Economy, health, pleasure \\
\hline
\end{tabular}

People decide how to travel according to a number of factors, including time spent on travel, availability, convenience, economy, and health. For example, high toll or parking fee might discourage people from driving, and traffic jams might encourage a potential car driver to take the bus. On the other hand, a traffic jam might also discourage a person from taking buses and encourage walking 
or biking instead. Even when people have decided to drive to work, there are still different options available, including sharing through carpool and selecting alternative routes.

\subsection{Behavioral Change}

Behavioral change is described in [8] through a behavioral change wheel (BCW), identifying sources of behavior, interventions, and policies. Sources of behavior include three components: capability, motivation, and opportunity [8]. Capability is defined as the individual's psychological and physical capacity to engage in the suggested activity. It includes having the necessary knowledge and skills. Motivation is defined as all those brain processes that energize and direct behavior. It also includes habitual processes, emotional responding, and analytical decision-making. Opportunity is defined as all the factors that lie outside the individual that make the behavior possible or not [8].

Motivation is influenced by both capability and opportunity, while all three components influence the behavior. For example, to nudge a person to ride a bike, a necessary capability is access to a bike and the skills to do so. It also requires the opportunity to bike. For example, if the distance is unreasonably long, the nudge should not be given.

Interventions described in $\mathrm{BCW}$ include functions that can be used to address deficits in one or more of the capabilities, opportunities, or motivation components. Examples of functions are education, training, persuasion, coercion, incentivization, and modeling. The $\mathrm{BCW}$ also identifies policies that are decisions made by authorities that support the delivery of intervention functions [8].

Nudging represents a new intervention function that can be added to BCW. A smart nudge will both provide practical information about the recommended activity, and motivating information that gently pushes the user towards the activity. Smart nudging, therefore, targets both motivation and capability to change behavior. Nudging in general (i.e., not restricted to digital/smart nudging) also involves the opportunity component. This implies that changes to the physical environment (e.g., road planning and building easily accessible cycling and walking paths) have a significant impact on individuals' choices and may count as a nudge.

\subsection{Ethics and Transparency in Nudging}

Nudging is about influencing people towards some behavioral change, without limiting people's freedom of choice. To consider nudging a benefit, we must assume that the nudging goal represents some common good, and that nudges are designed by a well-meaning party that has people's best interests in mind. However, this cannot always be assumed.

The ethics of nudging has been discussed and questioned, for example, by pointing to the potential danger of manipulating people and presenting unfair nudges [1,2,9]. In [2], the following is said about the ethics of nudges:

"All over the world, governments are using nudges as regulatory tools. Is this ethical? Much of the answer depends on whether nudges promote or instead undermine welfare, autonomy, and dignity. Many nudges, and those that deserve support, promote some or all of those ideals, and undermine none of them."

Transparency, where users are made aware that nudges try to influence them, is considered an important safeguard against manipulation and nudges that undermine the users' best interests [1,10-12]. A framework distinguishing between transparent and non-transparent nudges is described in [11]. This may be used for characterizing nudges and reason about their positive and negative effects. The research described in $[10,12]$ shows that transparent nudges may well be effective. This contradicts work that claim nudges work best in the dark [13].

Digital and smart nudging systems are typically provided through some application on a mobile device. Nudge transparency can, in these cases, be achieved by clearly stating the nudging goal as part of the application description. The user is then warned about the intention to nudge and can 
decide whether or not to use the application. Such a warning can also be given each time the user starts the application.

\section{Nudgy Recommendations}

We apply nudgy recommendations in different application areas using a variety of user interaction mechanisms. Currently, we are investigating two areas: more environmentally friendly transportation and a healthier lifestyle.

We try to achieve more environmentally friendly transportation by changing the behavior of the population when choosing the means of transportation (see Table 1). For example, we could present a notification on their smartphone or smartwatch with information making an environmentally friendly transportation choice easier for the user: "If you leave your house in 15 minutes and walk to the bus stop marked on this map, you catch bus 35 on-time and arrive at work in time for a coffee before your 9 am meeting. The weather is nice today — bring your sunglasses."

A healthier lifestyle could work similarly. Personalization is used to identify what motives the current user to be more active, and notifications triggering the user is sent in situations where the user can be influenced to choose to be active. For example, we could present a user with a notification reminding her about a group activity at the nearby gym that many of her friends usually attend: "There are a few open spots available at Gym X for the group-training session that your friends usually attend. It starts in 45 minutes — Should I book it for you? Kim has registered for this session."

\subsection{Recommendations and Nudge-Driven Filtering}

Recommender systems are software tools and techniques that provide suggestions for items that are most likely of interest to a particular user [14]. Item is the general term used to denote what the system recommends to users. Since recommendations are usually personalized, different users or user groups benefit from diverse, tailored suggestions [14]. Basic models for recommender systems include content-based, collaborative, knowledge-based, demographic, and hybrid filtering [14-16].

- Content-based filtering is a well-known technique, which tries to match users to items that are similar to what they have liked in the past. This similarity is based on comparing data in the user profile to item features, to identify items that are considered relevant to the user.

- Collaborative filtering uses ratings provided by multiple users to make recommendations. When enough information is available, recommendations to a user are based on information provided by those users that are considered to have the most in common with the targeted user.

- Knowledge-based filtering is based on explicitly specified user requirements. Instead of using historical rating or buying data, external knowledge bases and constraints are used to create the recommendation.

- Demographic filtering is based on the assumption that individuals with certain common properties (e.g., sex, age, and country) have shared preferences concerning the choice of items.

- Hybrid filtering is based on a combination of two or more of the above filtering techniques.

Recommendation analysis is often based on previous interaction between users and items since past interests and proclivities are often good indicators of future choices [16]. For example, a user that is interested in a historical documentary is more likely to be interested in another historical documentary or an educational program, rather than in an action movie. The goal of recommender systems is thus to suggest items that are within the user's current interest area.

The goal of nudging, on the other hand, is to suggest items or activities that support the nudging goal (e.g., healthier or more environmentally friendly behavior). This implies that the suggested item or activity is outside the main interest or regular activity of the user, and it is meant to push the user towards a change in behavior. Thus, for nudgy recommendations, none of the traditional recommender system models apply, since our goal is not to recommend the user to continue choosing 
what she usually does, but rather suggest moving on to something new and (in the light of the nudging goal) better.

We suggest $n u d g e-$ driven filtering, where activities (or items) are suggested based on a nudging goal that determines the scope and direction of the targeted behavior. The nudge is based on knowledge about what the user has chosen in the past, and the system selects an activity that on a scale better fulfills the nudging goal. For example, if the user typically drives a private car, the suggested activity could be using a bus or walking. Both these transportation means are more environmentally friendly compared to the car. Nudge-driven filtering is first about selecting a targeted activity, and second about selecting information that is useful for designing a nudge that the user may have a positive reaction to.

\subsection{Architecture}

High-level architecture for a smart nudging system is depicted in Figure 2. The nudging process involves a number of tasks that are seen in the figure as separate components.

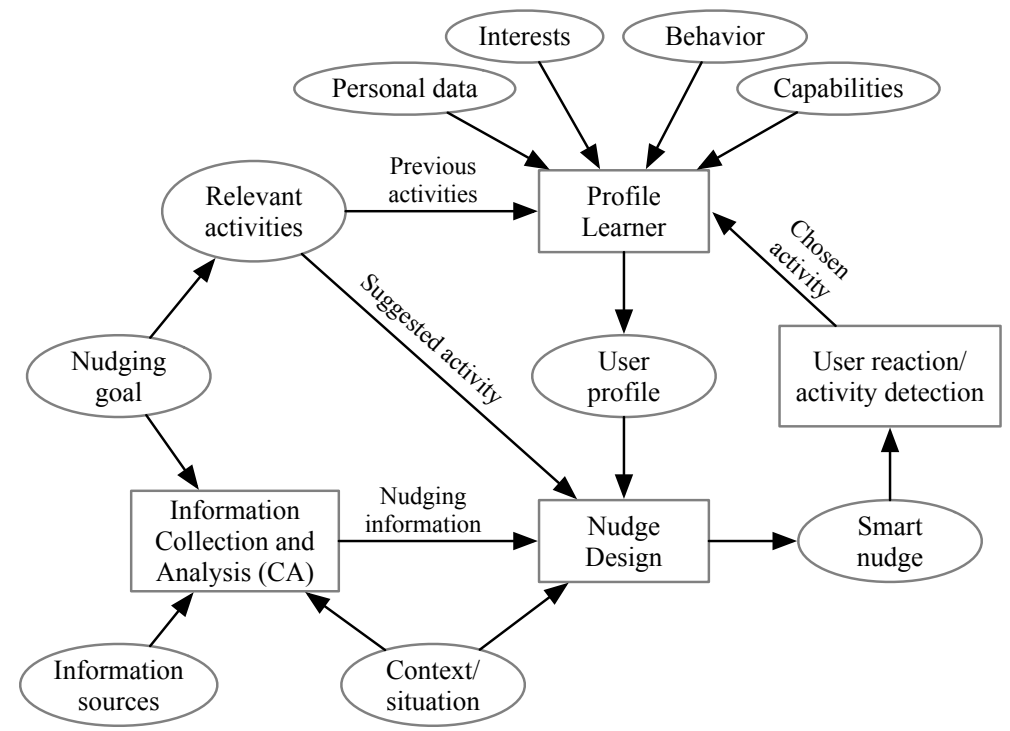

Figure 2. Architecture of a smart nudging system.

The nudging goal is central to the system in that it determines the relevant activities (or items) that can be recommended. The goal is also used as input to the information collection and analysis component since the goal is vital for determining supporting information to use in nudges.

To have smart nudging, where nudges are personalized to the needs and situation of a specific user, a user profile is important. The profile is created by the Profile Learner that collects information (such as age, gender, interests, and behavior) that describes and represents the user. The Profile Learner also monitors previously chosen activities to determine the user's preferred transportation choice(s) and user capabilities (for example if the user is currently capable of walking or cycling).

The Information Collection and Analysis (CA) component collects information that is used for creating the nudge. We call this nudging information, and it is used for providing practical information to users about the selected activity, as well as motivating the user to accept the nudge. This may include bus routes and departure time, road and footpath conditions, distance and time to destination, cost of traveling, weather conditions, and more. The component also collects user context information, for example, location, destination, calendar information (such as time and place for the next meeting) and opportunity for the activity. The collected information is combined, analyzed, and processed to produce the right set of information to motivate and inform about the selected activity.

The Nudge Design component creates personalized nudges that are relevant to the goal, and it is tailored to the specific user. It provides information that is useful for selecting the suggested activity. 
The nudge must also be timely and relevant concerning decisions that need to be taken by the user. The result of the nudge design is a smart nudge presented to the user.

The evaluation of the nudge includes monitoring of the user reaction to the nudge and identification of the activity chosen by the user. The effectiveness of the nudge is used as input to the Profile Learner and is it is reflected in the user profile. The nudge itself and the outcome are both stored and used as a knowledge base for further nudging.

\subsection{User Profile Learning}

In [7], we discussed personalization in the context of nudging. A smart nudging system uses personalization and context-awareness to design nudges that are relevant to the current situation of the user. Personalization requires a user profile that identifies interests, behavior, and other characteristics of the user. Typical information in a user profile is shown in Table 2 [17].

User profile information can be collected explicitly, through direct user participation, or implicitly, through automatic monitoring of user activities [18,19]. In explicit information gathering, the user herself provides information through for example specification of interests, and positive or negative responses to nudges. Implicit collection of profile information is a continuous process, where the current interests and behavior is continuously mined.

The user's preferred transportation means are important information for a smart nudging system focusing on green transportation choices. For a new user, this can initially be explicitly provided, while it later is implicitly gathered through activity monitoring. User interests, such as being fond of biking or skiing, can also be explicitly provided, or implicitly detected through for example search activity on the Internet.

Wearable sensors can provide health-related information about a user, such as heart rate, blood pressure or activity monitoring (for example, walking or sleeping pattern), while sensors monitoring the physical environment (such as temperature, noise, and air pollution measurements) provide information about the surrounding of the user.

The user profile must reflect how behavior changes over time, and, therefore, it includes historical information on the evolving of behavior. We also include nudging history in the user profile, to reflect which types of nudges have been given to the user, and how the user reacted to them. When designing new nudges, it is useful to know which types of nudging that previously have been positively received by the user.

Table 2. Main user profile dimensions.

\begin{tabular}{ll}
\hline Dimension & Description/examples \\
\hline Personal data & Gender, age, nationality and preferred language \\
Cognitive style & The way in which the user process information \\
Device information & May be used to personalise presentation of information \\
Context & The physical environment where the user processes information \\
History & The user's past interactions \\
Behaviour & The user's behaviour pattern \\
Interests & Topics the user is interested in \\
Intention/goal & Intention, goals or purposes of the user \\
Domain knowledge & The user's knowledge of a particular topic \\
Nudging history & The user's reactions to previous nudges \\
Capabilities & The user's psychological and physical capacity to engage in activities \\
\hline
\end{tabular}

\subsection{Information Collection and Analysis}

As we discussed in [7], the nudging system collects data that are historical, current, and represent plans and predictions. Historical data describe the past. Current data describe the current situation. 
Plans are data describing schedules or planned activities. Predictions are data trying to guess the future, often based on historical data, current data, and plans. Examples of historical data, current data, plans, and predictions are found in Table 3 [7].

Table 3. Examples of historical data, current data, plans and predictions (from [7]).

\begin{tabular}{ll}
\hline Data & Examples \\
\hline Historical & $\begin{array}{l}\text { Historical sensor data (weather/pollution); congestion history and traffic flow; successful and } \\
\text { unsuccessful user travel experiences; past events }\end{array}$ \\
Current & $\begin{array}{l}\text { Current sensor data (e.g., weather/pollution); road, footpath and ski-track conditions; current } \\
\text { pollution levels; current traffic conditions; current location of expected bus }\end{array}$ \\
Plans & $\begin{array}{l}\text { User's calendar events; bus and train schedules; planned infrastructure maintenance; } \\
\text { holidays/recreation days; festivals/events influencing transportation infrastructure } \\
\text { Predictions }\end{array}$ \\
& $\begin{array}{l}\text { The weather the rest of the day; predicted pollution levels during the day; the deviation from the } \\
\text { schedule of a bus leaving a nearby bus stop }\end{array}$ \\
\hline
\end{tabular}

Nudging information may come from sources such as IoT sensors, body sensors, online services, crowdsourcing, and crowdsensing. Sensors that are useful for transportation nudging are for example location and proximity sensors, which are used to track both users and vehicles, weather and environment sensors, and can influence the prediction of the next day's weather and pollution level. Sensors sensing traffic flow, congestions, footpath usage, available parking, etc. are available in many forms. Other sensors can detect the conditions of roads, footpaths, bike lanes, and ski tracks, and be used to detect for example slippery (e.g., icy) roads, shoveling status, and if the ski track has recently been prepared. These and other sources of information are described in more detail in [7].

Data from different sources are combined, and a variety of analysis, data mining, and statistical tools can be used to extract information from the raw data. A simple example is to use the current location of the user (provided by the personal smartphone) combined with the next event in her calendar, to prepare a notification about when to start traveling to reach the event on time. The event provides a location and a start time, while travel time, departure, route, and costs can be calculated based on information from online services.

To motivate the user, a nudge can, for example, target economic benefits, pleasure, or convenience. A nudge can thus include information such as "walking saves you 6 euro", "the weather is nice, why not walk?", "if you walk to work, you don't have to exercise in the afternoon", or "if you walk, you avoid the traffic jam". To provide such information, knowledge of the current situation, and comparison of different transportation alternatives are necessary.

To nudge for green transportation choices, the analysis performed involves a wide range of data combined with sophisticated modeling of transportation options and user behavior. The complexity of producing a good set of travel options for a user can consequently be relatively high.

\subsection{Nudge Design Approaches}

A smart nudge is designed for the suggested activity, using nudging information from the CA component together with knowledge of the user, provided through the user profile and context information. The Nudge Design component must determine what to nudge (which activity), which nudging information is useful for presenting the nudge, how often a nudge should be given, and at what time to present it.

Experiments show that nudging works particularly well when there are immediate, or at least short-term, benefits for the individual [20]. Thus, when designing nudges, identifying and presenting such immediate or short-term benefits would be valuable. Nudging for green transportation choices can have both short and long-term benefits. For example, in areas with much congestion, cycling to work may save both time and money. If more people choose the bicycle, long-term benefits are less air 
pollution, carbon dioxide emissions, reduced traffic, and thereby less congestion. Additionally, using the bicycle instead of driving represents a health benefit for the user.

A challenge of nudge design is how to present to the user the benefits of choosing the suggested activity. For example, how can a nudge convince the user, on a rainy day when the convenience of driving seems obvious and tempting to the user, that walking is a good choice?

Important approaches (or tools) for nudging in general (i.e., not focusing specifically on digital or smart nudging) are presented in $[9,21]$. These describe different ways nudging can influence an individual's behavior. The following list presents approaches from [21] that are particularly useful for digital and smart nudging, and it exemplifies them in the context of green transportation choices.

- Simplification and framing: This targets the way information (or the choice to be made) is presented to the user. Complexity and information overload cause confusion, and resistance to change need not be caused by disagreement or skepticism, but of perceived difficulty [21]. Thus, to encourage certain behavior, simplification can be helpful. Producing nudges that makes it easy to choose the suggested activity is therefore encouraged. People make decisions depending on how information is presented, and framing is the conscious phrasing of information to support the targeted behavior.

Example 1. Simplifying the task of taking public transportation to some unknown destination could include presenting the user with only the currently relevant information. Not the complete set of bus tables, possible bus changes and a lot of other options that might be available. In addition, providing the user with all necessary information for the suggested transportation relieves the user from collecting additional information herself. A nudge can, for example, include bus departure time, directions to the bus stop, guide to buying a ticket, travel time, information about the end bus stop and how to walk to the final destination.

- Default rules: People are greatly influenced by defaults (i.e., standard choices) that determine the result in case people take no action.

Example 2. Tourist information can present buses or walking paths as the default way to reach sights or stores, information about parking lots from where the buses depart, and present general transportation choices ordered by environmental friendliness.

- Use of social norms: Social norms influence human behavior, and individuals tend to orient towards the behavior of others, searching for social proof when unable to determine the appropriate mode of behavior in a given situation.

Example 3. A nudge can inform the user that "most people take the bus to work". One can also let users share experiences and tips regarding green transportation within social networks (e.g., share the current state of cycling and walking paths, personal achievements, and positive experiences), and provide competition among workplaces regarding the adoption of green transportation choices.

- $\quad$ Reminders: People tend to have a lot on their minds, and may forget, be preoccupied or simply postpone starting an activity. A reminder can be handy as a nudge for specific behavior.

Example 4. A nudge can remind the user to start the day sufficiently early to catch the bus or have time to walk to work. When finishing work, a reminder of the departure time of the bus, may get the user home in time for family activities.

- Informing people of the consequences of their own past choices: By continuously monitoring the user and her activities, information about a person's past choices is available and can be used for informing the user about the long-term effects of continuing this behavior. 
Example 5. This may be specifically useful when nudging for a healthier lifestyle. Informing a user about the positive effect of activities for her health may trigger the user to be more active.

These nudging approaches are based on psychological mechanisms that underlie nudging. An overview of such psychological effects is given in [22], where 20 effects are described. In addition to the above approaches, a couple of other relevant effects can be mentioned:

- Loss aversion: Loss aversion assumes that losses and disadvantages have a more significant impact on preferences than gains and advantages.

- Hyperbolic discounting: Hyperbolic discounting means that individuals behave inconsistently in terms of time. They value the present and the near-present stronger than the future. This supports that nudges presenting immediate or short-term benefits are more effective than nudges focusing on long-term benefits.

The timing of a smart nudge is vital for how the user receives it. First, the nudge must be presented at the time when a decision is to be made, giving the user sufficient time to act upon it. A nudge should also be presented sufficiently often to push the user continuously, but not so often that the user is annoyed. If a nudge for taking the bus is followed a number of times, this may indicate that the user has made some behavioral change and are now using public transportation. At this point, the nudge can be given less often or entirely stopped for a period, while monitoring user activity to determine if the bus continues to be used. On the other hand, if the user is repeatedly not following a nudge, this may indicate that the user is not ready for this particular behavioral change, and the nudge should stop or be replaced by a nudge for a different means of transportation.

\subsection{User Reaction and Activity Identification}

A considerable challenge for a smart nudging system is to determine which tool or type of nudge to use. The knowledge of psychological effects on behavior, applies to users in general, while individual reactions to a nudge are much more difficult to predict.

A smart nudge, created by the Nudge Design component, is presented to the user, who can choose to follow the nudge or ignore it. To determine the user reaction, our nudging system includes a component that monitors the user and detects the selected activity after a nudge is given. The findings from this component contribute to the knowledge about the user, and it is represented in the user profile.

By continuously monitoring the user, it is possible to detect preferred transportation means and whether the preference change over time. It can also detect if nudges seem to be followed, i.e., if the suggested activity, is the one that is selected by the user. The component can also record each nudge and the reaction to the nudge. By analyzing the nudge history for a user, one can classify nudges to uncover which types of nudging that seem to be effective for the specific user. User preferences concerning nudges may, however, be affected by many variables, such as time, location, social context and more.

\section{Processing}

To be able to make nudgy recommendations based on smart nudging, many data have to be collected, aggregated, combined, and processed. Data processing is an essential part of a smart nudging system, and, as discussed in [7], the processing can happen on dedicated servers, in a distributed fashion, at the data source, on the user devices, and in any combination of this. Processing is done to prepare nudgy recommendations and to maintain and update user profiles.

The decision on where to process data is based on what kind of analytics are performed (including the complexity of the analytics and data necessary to perform the analytics), the sensitivity of the data (e.g., personal data and health data), and the timing and characteristics of the usage of the data. Processing at dedicated servers or in a distributed fashion could support more complex analytics with larger datasets, but users might not agree to share personal and private information with such 
servers (and it might not comply with privacy regulations). Performing the analytics on the personal user device (the smartphone of the user) could ensure that personal and private information never leaves the user device, but it might limit the complexity of the analytics performed and limit the data available for the task.

\section{User Behavior and Privacy Preserving Processing}

In smart nudging, one goal is to improve nudging based on the observed behavior of the user. For example, based on the successful and unsuccessful nudging of current users, new knowledge is aggregated that can be used to improve future attempts to nudge the user. This aggregated knowledge could also be used to improve the nudging of other users, using a collaborative-filtering type of approach. When nudging a specific user, behavior selected for a similar context by users with matching profiles could identify activities that would also be acceptable for the current user.

The problem is that this knowledge is based on personal and private data that, for privacy concerns, should not be shared. If the data of the current user were going to be used to improve the nudging of other users, they would have to be used in a way that ensures the privacy of the current user. We have to do privacy preserving processing of the data.

Different techniques, including anonymization and obfuscation of data, secure multiparty computation, and differential privacy, can be used to achieve this. The details of these approaches are out of the scope of this article and are discussed in more detail in [23].

\section{Related Work}

\subsection{Interventions for Behavioral Change}

Smart nudging represents an intervention function for behavioral change that can be added to the behavioral change wheel, described in Section 2.3. In Table 4, we compare the intervention functions from the original wheel [8] with smart nudging, by linking interventions to the capability, opportunity, and motivation components.

Smart nudging is different from the other interventions in Table 4, in that it both informs (through practical information) and gently pushes (through motivation) towards the recommended activity. It thus links to psychological capability, as well as reflective and automatic motivation.

Table 4. Comparison of intervention functions for behavioral change, with links to capability, opportunity and motivation (links for all interventions except smart/digital nudging is from [8]).

\begin{tabular}{|c|c|c|c|c|c|c|}
\hline & $\begin{array}{c}\text { Physical } \\
\text { Opportunity }\end{array}$ & $\begin{array}{c}\text { Social } \\
\text { Opportunity }\end{array}$ & $\begin{array}{c}\text { Physical } \\
\text { Capability }\end{array}$ & $\begin{array}{c}\text { Psychological } \\
\text { Capability }\end{array}$ & $\begin{array}{l}\text { Automatic } \\
\text { Motivation }\end{array}$ & $\begin{array}{c}\text { Reflective } \\
\text { Motivation }\end{array}$ \\
\hline $\begin{array}{l}\text { Smart/digital } \\
\text { nudging }\end{array}$ & & & & $x$ & $x$ & $X$ \\
\hline Education & & & & $x$ & $x$ & \\
\hline Persuasion & & & & & $x$ & $x$ \\
\hline Incentivation & & & & & $x$ & $x$ \\
\hline Coersion & & & & & $x$ & $x$ \\
\hline Training & & & $x$ & $x$ & & \\
\hline Restriction & $x$ & $X$ & & & & \\
\hline $\begin{array}{l}\text { Environmental } \\
\text { restructuring }\end{array}$ & $x$ & $x$ & & & $x$ & \\
\hline Modelling & & & & & $x$ & \\
\hline Enablement & $x$ & $x$ & $x$ & $x$ & $x$ & \\
\hline
\end{tabular}




\subsection{Nudge vs. Persuasive Technology}

Digital nudging [24] and persuasive technology [25] should be compared as they both aim at persuading or nudging people to make some changes. However, persuasion is very often about human-computer interaction and how to present information or possible choices to the user. For smart/digital nudges, we also focus on information integration to provide the right combination of information to make it easier for the user to make, for him or her, non-obvious behavioral choices. This difference is also shown in Table 4, where smart nudging links to both capability and motivation components, while persuasion only links to motivation.

In [3], nudging and digital nudging is compared with persuasive technology. Based on their definitions, the two approaches are different on restrictions, in that nudging must be done "without forbidding any options or significantly changing their economic incentives", while persuasive technology must be done "without using coercion or deception". This implies that persuasive technology actually can limit the user's choices, while it is imperative for nudging to keep all options open.

\subsection{Nudging in the Transportation Domain}

Different experiments of nudging in the personal transportation domain are described and analyzed in [9]. Most of these approaches do not use digital nudging, but rather focus on other aspects of nudging. Experiments on simplification and framing of information include decluttering streets, maps to encourage cycling and walking, offering cycling training, or personal travel plans. Changing the physical environment includes road planning with lines, colors, signs and humps, separate cycling lanes, and sufficient parking space for bikes. There have also been experiments on smartphone apps to encourage physical activity and travel or walking feedback programs where social norms and social networks are involved [9].

The work in [26,27], which focuses on recommender systems as a tool for nudging users towards eco-friendly traveling decisions, is closer to our work. The proposed travel recommender system filters and ranks the available trip choices by considering user preferences and contextual elements while trying to balance user perceived route utility and $\mathrm{CO}_{2}$ emissions. In [27], the system provides a pool of messages that can be used for persuading the users. Compared to our work, the main difference is our focus on smart nudging and personalization with the collection of a wide range of data and data types, the integration and processing of these data, and the continuously implicit updating of the users' user profiles.

\section{Discussions}

Smart nudging provides recommendations that aim at gently pushing the user towards some overall nudging goal, such as choosing "more environmentally friendlier transportation".

General guidelines or tools for designing nudges are based on psychological mechanisms and knowledge on how people, in general, make decisions. However, there are individual differences, where two persons need not react in the same way to a specific nudge. The reaction may depend on factors such as time, location, situation, capability, personality, preferences concerning intervention, and more. Since so many factors may influence a user decision, determining how a personalized smart nudge is to be presented is a considerable challenge. Nevertheless, to provide effective nudging and facilitate behavioral change, it is necessary to personalize nudges to fit the reaction of the specific user.

Successful nudging requires flexibility concerning the selection of both activities to nudge for, and the accompanying information. If the user repeatedly accepts a specific activity through nudges, this might indicate a behavioral change, and the system should adapt so that this activity is less recommended (to avoid annoying the user). As the user slowly improves her behavior (according to the nudging goal), nudges must evolve to target new activity or behavior that fulfills the nudging goal even better. This is done to make the user reach to improve her behavior continually. If the nudge for a specific activity is continuously unsuccessful, further nudges should be designed differently, by 
choosing a different activity to nudge, presenting the nudge using different information, changing the timing, or choosing another nudging approach or tool. Making such adjustments are challenging and require reasoning concerning user reaction to previous nudges.

According to the definition of nudging, we cannot remove any options when nudging people. This means that we cannot limit the users' choices and must allow the user to choose other options than the selected activity. Therefore, the nudging system must also provide information concerning activities other than the one that is targeted in the nudge. The system can design a nudge that focuses on, for example, using the bicycle to work and collect information to support that. However, if the user instead would like to take the bus, the system should also provide useful information about this option.

Nudging for green transportation choices has a set of possible activities (or items) that is much smaller than in most recommender systems (e.g., systems for recommending books, music, and movies), where the number of available items is enormous. Choosing transportation can in many cases be reduced to choosing between the use of the private car, carpooling, public transportation (bus, train, subway), cycling, walking, skiing, and a few more. However, the complicating factor for nudging compared to recommender systems is the collection, selection, and combination of supporting information that is needed for designing the nudge. Adapting the nudge to reflect positive or negative reactions to previous nudges is also a complicated task. It involves monitoring the user's behavior, identifying trends, find alternative nudging approaches, and updating the user's user profile.

\section{Conclusions}

In this paper, an approach for recommender systems based on nudging has been presented. The focus has been on digital nudging tailored to the current situation of individual users. We call this smart nudging. Our main contributions are how to create a smart nudge, an architecture for a smart nudging system, and the personalization of nudges.

Author Contributions: Conceptualization, R.K. and A.A.; methodology, R.K. and A.A.; software, R.K. and A.A.; investigation, R.K. and A.A.; writing-original draft preparation, R.K. and A.A.; writing-review and editing, R.K. and A.A.; visualization, R.K. and A.A.; and project administration, R.K. and A.A.

Funding: Telenor contributed to fund equipment at our laboratory.

Acknowledgments: We would like to thank all people involved in the NUDGE project and our smart lab activities. Thank you to our colleague in the Open Distributed Systems research group, Weihai Yu, for his contributions on green transportation. Thank you to Arne Much-Ellingsen from Telenor for providing insight and a long-time collaboration with all things mobile. Thank you to the students Øysten Tveito, Thomas Holden, Pontus Aurdal, Maren Sofie Ringsby, Fredrik Høisæther Rasch, Håkon Wallann, Lady Limunga Jemea, Cosmin Radu Craciun and Sandor Dalecke, for experimenting and documenting all things IoT at our laboratory. Thank you to Telenor for long time cooperation and their support in producing the experiments needed to produce this text. Thank you to the technical staff at our department, Ken-Arne Jensen, Jon Ivar Kristiansen and Kai-Even Nilssen, for facilitating our laboratory and experiments. We also have to thank Michael Morreau and Erik Lundestad at the Department of Philosophy for interesting discussions regarding nudging, crowdsourcing, and much much more. Finally, we would like to thank all students at the Inf-3910-3 course in Spring 2017, Spring 2018, and Spring 2019 for experimenting with microcontrollers, sensors, LoRaWAN/NB-IoT networks, and back-end and front-end systems.

Conflicts of Interest: The authors declare no conflict of interest.

\section{References}

1. Thaler, R.H.; Sunstein, C.R. Nudge: Improving Decisions about Health, Wealth, and Happiness; Yale University Press: New Haven, CT, USA, 2008.

2. Sunstein, C.R. The ethics of nudging. Yale J. Reg. 2015, 32, 413-450. [CrossRef]

3. Meske, C.; Potthoff, T. The DINU-model-A process model for the design of nudges. In Proceedings of the 25th European Conference on Information Systems (ECIS), Guimarães, Portugal, 5-10 June 2017; pp. 2587-2597. 
4. Schneider, C.; Weinmann, M.; vom Brocke, J. Digital Nudging: Guiding Online User Choices Through Interface Design. Commun. ACM 2018, 61, 67-73, doi:10.1145/3213765. [CrossRef]

5. Commission of the European Communities. Towards a New Culture for Urban Mobility; Green Paper COM(2007) 551 final, European Commission: Brussels, Belgium, 2007.

6. United States Environmental Protection Agency. Greenhouse Gas Emissions from a Typical Passenger Vehicle. Available online: http:/ /biketoworkottawa.ca/wp-content/uploads/2018/03/greenhouse-gasemissions.pdf (accessed on 6 June 2019)

7. Andersen, A.; Karlsen, R.; Yu, W. Green transportation choices with IoT and smart nudging. In Handbook of Smart Cities: Software Services and Cyber Infrastructure; Maheswaran, M., Badidi, E., Eds.; Springer: Cham, Switzerland, 2018; pp. 329-352, doi:10.1007/978-3-319-97271-8.

8. Michie, S.; Van Stralen, M.M.; West, R. The behaviour change wheel: A new method for characterising and designing behaviour change interventions. Implement. Sci. 2011, 6, 42. [CrossRef] [PubMed]

9. Lehner, M.; Mont, O.; Heiskanen, E. Nudging-A promising tool for sustainable consumption behaviour? J. Clean. Prod. 2015, 134, 166-177, doi:10.1016/j.jclepro.2015.11.086. [CrossRef]

10. Bruns, H.; Kantorowicz-Reznichenko, E.; Klement, K.; Jonsson, M.L.; Rahali, B. Can nudges be transparent and yet effective? J. Econ. Psychol. 2018, 65, 41-59. [CrossRef]

11. Hansen, P.G.; Jespersen, A.M. Nudge and the Manipulation of Choice. Eur. J. Risk Regul. 2013, 4, 3-28. [CrossRef]

12. Loewenstein, G.; Bryce, C.; Hagmann, D.; Rajpal, S. Warning: You are about to be nudged. Behav. Sci. Policy 2015, 1, 35-42. [CrossRef]

13. Bovens, L. The ethics of nudge. In Preference Change; Springer: Berlin, Germany, 2009; pp. $207-219$.

14. Ricci, F.; Rokach, L.; Shapira, B. Recommender systems: Introduction and challenges. In Recommender Systems Handbook; Springer: Boston, MA, USA, 2015; pp. 1-34.

15. Bobadilla, J.; Ortega, F.; Hernando, A.; Gutiérrez, A. Recommender systems survey. Knowl.-Based Syst. 2013, 46, 109-132. [CrossRef]

16. Aggarwal, C.C. Recommender Systems; Springer: Berlin, Germany, 2016.

17. Gao, M.; Liu, K.; Wu, Z. Personalisation in Web Computing and Informatics: Theories, Techniques, Applications, and Future Research. Inf. Syst. Front. 2010, 12, 607-629, doi:10.1007/s10796-009-9199-3. [CrossRef]

18. Gauch, S.; Speretta, M.; Chandramouli, A.; Micarelli, A. User Profiles for Personalized Information Access. In The Adaptive Web; Brusilovsky, P., Kobsa, A., Nejdl, W., Eds.; Springer: Berlin, Germany, 2007; Volume 4321, pp. 54-89, doi:10.1007/978-3-540-72079-9\_2.

19. Ghorab, M.; Zhou, D.; O'Connor, A.; Wade, V. Personalised Information Retrieval: Survey and classification. User Model. User-Adapt. Interact. 2013, 23, 381-443, doi:10.1007/s11257-012-9124-1. [CrossRef]

20. Mont, O.; Lehner, M.; Heiskane, E. Nudging: A Tool for Sustainable Behaviour?; Report 6643; The Swedish Environmental Protection Agency (Naturvårdsverket): Stockholm, Sweden, 2014.

21. Sunstein, C.R. Nudging: A very short guide. J. Consumer Policy 2014, 37, 583-588. [CrossRef]

22. Mirsch, T.; Lehrer, C.; Jung, R. Digital nudging: Altering user behavior in digital environments. In Proceedings of the Internationalen Tagung Wirtschaftsinformatik (WI 2017), St. Gallen, Switzerland, 14 February 2017; pp. 634-648.

23. Andersen, A.; Karlsen, R. Privacy preserving personalization in complex ecosystems. In Digital Marketplaces Unleashed; Linnhoff-Popien, C.; Schneider, R.; Zaddach, M., Eds.; Springer: Berlin, Germany, 2017; pp. 247-261, doi:10.1007/978-3-662-49275-8.

24. Weinmann, M.; Schneider, C.; vom Brocke, J. Digital Nudging. Bus. Inform. Syst. Eng. 2016, 58, 433-436, doi:10.1007/s12599-016-0453-1. [CrossRef]

25. Fogg, B.J. Persuasive technology: Using computers to change what we think and do. Ubiquity 2002, 2002, 89-120. [CrossRef]

26. Bothos, E.; Apostolou, D.; Mentzas, G. Recommender systems for nudging commuters towards eco-friendly decisions. Intell. Decis. Technol. 2015, 9, 295-306. [CrossRef] 
27. Bothos, E.; Apostolou, D.; Mentzas, G. A Recommender for Persuasive Messages in Route Planning Applications. In Proceedings of the 7th International Conference on Information, Intelligence, Systems and Applications (IISA 2016), Chalkidiki, Greece, 13-15 July 2016, doi:10.1109/IISA.2016.7785399.

(C) 2019 by the authors. Licensee MDPI, Basel, Switzerland. This article is an open access article distributed under the terms and conditions of the Creative Commons Attribution (CC BY) license (http:/ / creativecommons.org/licenses/by/4.0/). 